\title{
Spotlight on siponimod and its potential in the treatment of secondary progressive multiple sclerosis: the evidence to date
}

This article was published in the following Dove Press journal:

Drug Design, Development and Therapy

2 November 2017

Number of times this article has been viewed

\author{
Alberto Gajofatto \\ Department of Neuroscience, \\ Biomedicine and Movement Sciences, \\ University of Verona, Verona, Italy
}

\begin{abstract}
Siponimod (BAF312) is a synthetic molecule belonging to the sphingosine-1-phosphate (S1P) modulator family, which has putative neuroprotective properties and well-characterized immunomodulating effects mediated by sequestration of B and T cells in secondary lymphoid organs. Compared to fingolimod (ie, precursor of the S1P modulators commercially available for the treatment of relapsing-remitting [RR] multiple sclerosis [MS]), siponimod exhibits selective affinity for types 1 and $5 \mathrm{~S} 1 \mathrm{P}$ receptor, leading to a lower risk of adverse events that are mainly induced by S1P3 receptor activation, such as bradycardia and vasoconstriction. In addition, S1P1 and S1P5 receptors are expressed by neurons and glia and could mediate a possible neuroprotective effect of the drug. A Phase II clinical trial of siponimod for RR MS showed a significant effect of the active drug compared to placebo on reducing gadolinium-enhancing lesions on brain magnetic resonance imaging (MRI) after 3 months of treatment. In a recently completed Phase III trial, treatment with siponimod was associated with a significant reduction in disability progression in secondary progressive (SP) MS patients compared to placebo. In this article, current evidence supporting siponimod efficacy for SP MS is reviewed.
\end{abstract}

Keywords: multiple sclerosis, siponimod, progression, disability, treatment

\section{Background}

Secondary progressive (SP) multiple sclerosis (MS) is a clinical subtype of the disease characterized by continuous worsening of neurological disability that begins insidiously in patients with a preceding relapsing-remitting (RR) course, which is the mode of onset of $\sim 90 \%$ of cases. The risk and timing of transition from RR MS to SP MS are highly unpredictable in the individual MS patient; however, it is estimated that up to $90 \%$ of RR MS cases may evolve to SP MS over a 25-year time interval. ${ }^{1}$ While patients with RR MS tend to recover fully or partially after relapses and may experience mild neurological symptoms, SP MS cases usually have gradual - although not necessarily steady - accumulation of neurological disability, typically affecting walking, sphincter control, hand function, speech, swallowing, and cognition. In addition, several disease-modifying therapies (DMTs) have shown efficacy in reducing relapses and overall disease activity in RR MS, while the majority of these and other investigational treatments have failed to prevent disease worsening in progressive MS. ${ }^{2}$ This may be explained by the predominance of acute inflammation, which is the main biological target of available MS DMTs, in the pathophysiology of RR MS clinical manifestations, ie, relapses that are known to decrease in frequency or even cease after the transition to an SP course. However, in the pathological continuum
Correspondence: Alberto Gajofatto Department of Neuroscience, Biomedicine and Movement Sciences, University of Verona, Piazzale LA Scuro 9, 37I 34 Verona, Italy

$\mathrm{Tel}+39458124768$

Fax +39458027492

Email alberto.gajofatto@univr.it (c) (1) (9) 2017 Gajofatto. This work is published and licensed by Dove Medical Press Limited. The full terms of this license are available at https://www.dovepress.com/terms.php

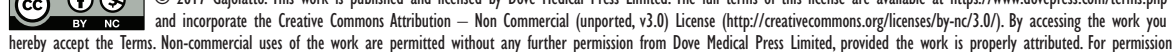
hereby accept the Terms. Non-commercial uses of the work are permitted without any further permission from Dove Medis
for commercial use of this work, please see paragraphs 4.2 and 5 of our Terms (https://www.dovepress.com/terms.php). 
between RR MS and SP MS, other factors may play a role, such as the effect of longstanding inflammation; exhaustion of compensatory and reparative mechanisms; and genetic, age-dependent, and exogenous factors. ${ }^{3}$ Approved DMTs for MS have little or no effect on the regulation of these biological processes as demonstrated by the modest or absent benefit of most treatments on disability progression that is independent of relapses.

Recently, a large Phase III placebo-controlled trial of the investigational agent siponimod for SP MS met its primary efficacy end point, showing a statistically significant reduction in confirmed disability progression in patients receiving the experimental intervention. ${ }^{4}$ The present article reviews the characteristics and development of siponimod and discusses its potential for future clinical use in MS.

\section{“Discovery” of BAF3 I 2 (siponimod)}

Siponimod (BAF312) is a structural analog of sphingosine, which is an endogenous sphingolipid involved in the regulation of a variety of biological functions, including lymphocyte trafficking, cardiomyocyte function, vascular development, and cell survival. ${ }^{5}$ Sphingosine-1-phosphate (S1P) is the bioactive metabolite of sphingosine in vivo, which binds to a family of G-protein-coupled receptors consisting of five distinct subtypes (S1PR1-S1PR5) expressed differentially on the surface of several cell types, eg, myocytes (S1PR1 and S1PR3), lymphocytes (S1PR1), astrocytes, oligodendrocytes, and neurons (S1PR1 and S1PR5). Of importance for immune system regulation, interaction of S1P with S1PR1 is essential for the egression of a subset of lymphocytes from secondary lymphoid organs and their redistribution to other tissues. The discovery that a chemically modified natural sphingolipid (ie, fingolimod) exhibits immunosuppressive effects after in vivo phosphorylation, by functionally antagonizing S1PR1 and thus sequestrating B and T cells in lymphoid tissues, led to the clinical development and approval of this molecule for the treatment of RR MS. ${ }^{6}$ However, fingolimod also has affinity for other S1PR subtypes, including S1PR3 that is internalized by cardiac myocytes after interaction with the phosphorylated form of the drug, leading to heart rate reduction, and rarely to atrioventricular block and cardiac arrest, particularly in the first 24 hours after treatment start. It is therefore recommended to perform 6-hour cardiac monitoring in all patients initiating fingolimod. In addition, fingolimod has a prolonged half-life in human beings requiring $\sim 2$ months for elimination after treatment interruption. These limitations prompted the pharmaceutical industry to develop S1PR modulators with shorter half-life and lacking affinity for S1PR3 with the objective of combining fingolimod efficacy for MS with a better pharmacokinetic and safety profile.

Siponimod is a synthetic derivative of fingolimod obtained with modifications providing increased rigidity in the lipophilic alkyl chain, which confers selectivity for S1PR1 and SIPR5 as shown in vitro. ${ }^{7}$ In vivo preclinical studies showed that siponimod was safe and significantly ameliorated symptoms of the murine animal model of MS (ie, experimental autoimmune encephalitis [EAE]), ${ }^{8}$ opening the way to the development of clinical trials of BAF312 for patients with MS.

\section{Siponimod clinical development for MS}

An exploratory Phase I trial of siponimod showed that drug administration was overall safe at multiple doses in a group of 48 healthy volunteers, although it induced transient (day 1 only) bradycardia despite sparing of S1PR3. In fact, BAF312 activates GIRK channels in human atrial myocytes likely through interaction with S1PR1, causing bradycardia. ${ }^{8}$ Pharmacokinetic analysis revealed that siponimod half-life is 30 hours with complete drug elimination in $\sim 7$ days. Following these findings, a dose-ranging randomized Phase II trial assessed the safety and efficacy of BAF312 vs placebo (BOLD study) in two independent cohorts of patients with RR MS enrolled in 72 centers. ${ }^{9}$ Subjects in cohort $1(\mathrm{~N}=188)$ received once daily siponimod $10 \mathrm{mg}, 2 \mathrm{mg}, 0.5 \mathrm{mg}$, or placebo (1:1:1:1) for 6 months. After a 3-month interim analysis, 109 additional patients were allocated to once daily siponimod $1.25 \mathrm{mg}, 0.25 \mathrm{mg}$, or placebo (4:4:1) for 3 months (cohort 2). Patients in cohort 1 immediately received the full siponimod dose, while in cohort 2, a masked titration scheme was implemented for treatment initiation. The primary outcome was the dose-response relationship of the five doses of siponimod compared with placebo during 3 months of treatment, based on the number of new gadoliniumenhancing lesions on T1-weighted images and new or newly enlarged non-enhancing lesions on T2-weighted monthly brain magnetic resonance imaging (MRI) scans. Secondary end points included also clinical efficacy measures, such as annualized relapse rate and proportion of relapse-free patients, and safety/tolerability data. Siponimod doses of $10,2,1.25$, and $0.5 \mathrm{mg}$ significantly reduced the number of new brain lesions (as defined in the primary end point) at 3 months compared to placebo with a dose-dependent relation. In addition, siponimod $2 \mathrm{mg}$ significantly reduced the annualized relapse rate at 6 months compared to placebo. 
A dose-dependent decrease in heart rate was observed during treatment initiation on day 1 , reaching nadir at $\sim 3$ hours after dosing and returning to levels similar to placebo in the following 24 hours in all treatment groups. Dose titration (siponimod $1.25 \mathrm{mg}$ group) mitigated heart rate reduction compared to full-dose administration on day 1. First- and second-degree atrioventricular blocks were reported in nine patients receiving siponimod 10,2 , or $0.5 \mathrm{mg}$. As expected, there was a dose-dependent total lymphocyte count decrease by $20 \%-74 \%$ at day 7 of siponimod treatment compared to baseline, which remained stable up to 3 and 6 months. Increased alanine aminotransferase concentrations were reported more frequently in the siponimod groups compared to placebo. In the 24-month extension of the trial, patients receiving siponimod continued with the originally allocated dose, while patients on placebo were randomized at baseline to one of the five siponimod doses..$^{10}$ The incidence of adverse events was similar across treatment groups. Nine out of 252 enrolled patients had serious adverse events, but no new or unexpected safety concerns emerged compared to the core BOLD study. The number of gadolinium-enhancing T1 lesions and new T2 lesions on brain MRI and the annualized relapse rates remained low over 24 months, particularly with the three or four highest siponimod doses.

In 2012, a large randomized, placebo-controlled, doubleblind Phase III trial of siponimod for SP MS (EXPAND study) was started in 31 countries. ${ }^{4}$ Eligible patients were aged 18-60 years, had progressive increase in disability for at least 6 months in the absence or independent of relapses, and a baseline Expanded Disability Status Scale (EDSS) score from 3.0 to 6.5 . The primary efficacy outcome was time to 3-month confirmed disability progression measured by EDSS. The key secondary outcomes were time to confirmed worsening of $\geq 20 \%$ from baseline in the timed 25 -foot walk test and brain $\mathrm{T} 2$ lesion volume change from baseline. The study enrolled 1,651 patients (60\% women) who were randomized 2:1 to receive either siponimod $2 \mathrm{mg}$ daily (with initial 6-day dose titration starting at $0.25 \mathrm{mg}$ ) or placebo for a variable duration determined by the time at which a prespecified number of patients reached the primary outcome in the overall study population. At baseline, mean age was 48 years, median EDSS score was 6.0, and $75 \%$ of patients had no gadolinium-enhancing T1 lesions. In addition, relapses in the 2 years prior to study start were documented in $35 \%$ of patients. ${ }^{11}$ Of the 1,651 included patients, 1,363 (83\%) completed the study. Median time on study was 21 months, with the majority of patients $(87 \%)$ participating for at least 1 year. Overall, there were
449 confirmed disability progression events. Treatment with siponimod was associated with $21 \%$ reduction in the risk of disability progression confirmed at 3 months ( $26 \%$ at 6 months) compared to placebo (hazard ratio $=0.79$, $p=0.013$ and hazard ratio $=0.74, p=0.006$, respectively). Accordingly, siponimod treatment showed a beneficial effect on other secondary efficacy outcomes, including the annualized relapse rate, $\mathrm{T} 2$ lesion volume, new T2 lesions, and number of gadolinium-enhancing lesions $(p<0.0001$ for all comparisons vs placebo). In addition, a benefit on percent brain volume change was also reported in patients receiving siponimod ( $p=0.0002$ vs placebo). Siponimod did not show a significant benefit on ambulation impairment as measured by timed 25 -foot walk. Adverse events in the siponimod group occurred at a similar rate (88.7\%), to that observed in the previous BOLD RR MS trial, leading to treatment discontinuation in $7.6 \%$ of patients. Most common adverse events $(>10 \%)$ were headache, nasopharyngitis, urinary tract infection, falls, and hypertension. Serious adverse events occurred in $17.9 \%$ of patients on siponimod and $15.2 \%$ of patients receiving placebo. Incidence of deaths $(0.4 \%$ vs $0.7 \%)$, malignancies ( $1.9 \%$ vs $2.6 \%)$, and infections (49.0\% vs $49.1 \%)$ was similar between treatment groups. Adverse events of interest included bradyarrhythmias (3.5\% vs $2.4 \%$ ), hypertension ( $12.6 \%$ vs $9.3 \%$ ), and macular edema $(1.8 \%$ vs $0.2 \%)$. Lymphopenia $<0.2 \times 10^{9} / \mu \mathrm{L}$ was observed in $2.7 \%$ vs $0.2 \%$ of patients, and liver function test elevation was three or more times greater than upper normal limit in $5.6 \%$ vs $1.5 \%$ of patients. ${ }^{12}$

\section{Discussion - insights into potential siponimod efficacy for SP MS}

SP MS is characterized by irreversible accrual of disability over time as a consequence of insidious worsening of neurological symptoms rather than failure of relapse recovery. This type of MS course is lacking a substantially effective treatment to reduce disability progression independent of relapses. The results reported for the siponimod Phase III SP MS study (ie, the largest clinical trial for SP MS ever) are encouraging, although the final data have still to be published in a full peer-reviewed paper. Based on available information, siponimod showed a statistically significant effect on reducing confirmed EDSS progression over $\sim 2$ years of treatment on average compared to placebo. This finding was consistent with the reduction in the relapse rate during the trial and the effect favoring siponimod on several MRI measures, including brain atrophy, new/enlarging T2 lesions, and gadoliniumenhancing lesions. However, there was a discrepancy with the 
data observed for a key secondary clinical end point, ie, gait speed measured by timed 25-foot walk, on which treatment with siponimod did not show a significant benefit. Although the full data analysis is not published yet, the magnitude of siponimod effect on the primary end point of the EXPAND trial (ie, 21\% reduction of 3-month confirmed EDSS score progression compared to placebo) appears robust and suggests a possible clinically meaningful benefit of the drug, particularly because of the scarcity of effective therapies for SP MS patients.

Currently approved DMTs for SP MS include interferon beta- $1 \mathrm{~b}$ and mitoxantrone, based on the results of two clinical trials that showed some benefits of these drugs on disability progression compared to placebo. In the trial conducted by the European Study Group on interferon beta-1b in SP MS, patients treated with interferon showed significantly less EDSS progression that was confirmed at 3 months compared to patients receiving placebo over 3 years (39\% vs 50\%). ${ }^{13}$ In the trial of mitoxantrone for rapidly progressive MS, patients on active drug had a stable or improved EDSS score at 2 years in a significantly higher proportion than patients treated with placebo (mean EDSS change -0.13 vs +0.23). ${ }^{14}$ Both studies recruited an "active" SP MS population, characterized by a high pretrial relapse rate and/or presence of gadolinium-enhancing lesions on baseline brain MRI. Post hoc analyses demonstrated that most of the effects on disability progression observed with interferon beta and mitoxantrone were actually driven by the significant relapse rate reduction determined by the active drugs rather than by halting disability progression per se. In fact, other trials of interferon beta-1a and $-1 \mathrm{~b}$ enrolling SP MS patients with lower prestudy relapse rate failed to show a significant benefit on EDSS progression. ${ }^{15}$ Similarly, several additional clinical trials were not able to consistently show a significant benefit of immunomodulatory and immunosuppressive drugs on disability progression in SP MS patients, with few exceptions. ${ }^{16}$ Among therapies not directly targeting the immune system, high-dose biotin (MD1003) has been recently investigated in a Phase III trial that enrolled 154 patients with SP or primary progressive (PP) MS and met its innovative primary end point, ie, the proportion of patients with disability reversal at month 9 , confirmed at month 12 , defined as an EDSS decrease of $\geq 1$ point ( $\geq 0.5$ for baseline EDSS 6-7) or a $\geq 20 \%$ decrease in timed 25 -foot walk. ${ }^{17}$ In this study, $13 \%$ of patients (mostly SP MS cases) treated with MD1003 reached the primary efficacy outcome compared to none of the patients receiving placebo. Interestingly, $>40 \%$ of subjects in the MD1003 group were on a concomitant DMT, suggesting that high-dose biotin may be safely administered in progressive MS patients as add-on treatment to immunomodulatory or immunosuppressive drugs.

Different from most previous positive SP MS studies, the EXPAND siponimod trial design and inclusion criteria allowed the enrollment of a widely representative SP MS population, characterized by evidence of disease progression with low relapse rate prior to study initiation and low proportion of gadolinium-enhancing lesions on baseline brain MRI. In this view, it seems unlikely that the effect observed on disability progression could be explained purely by relapse rate reduction and/or amelioration of disease processes reflected by MRI activity. In vitro and animal model studies have shown that, beside the immunomodulatory effects driven by lymphocyte trafficking regulation, siponimod may have protective properties against demyelination and neurodegeneration. O'Sullivan et $\mathrm{al}^{18}$ demonstrated that BAF312 activates pERK, pAKT, and calcium signaling (ie, molecular pathways involved in the regulation of cell survival and proliferation) in cultured mouse and human astrocytes, mainly through interaction with S1PR1. In the same study, treatment with siponimod attenuated demyelination induced in organotypic slice cultures prepared from mouse cerebellum. Recently, Gentile et $\mathrm{al}^{19}$ showed that intracerebroventricular infusion of siponimod in mice with EAE rescued defective GABAergic transmission and prevented GABAergic interneuron loss, which are at the basis of an inflammation-dependent synaptopathy possibly contributing to neurodegeneration and irreversible disability accumulation in demyelinating disease models. Furthermore, the investigators reported reduced release of IL-6 and RANTES from activated microglial cells in vitro after BAF312 treatment, in addition to astrogliosis and microgliosis reduction together with lymphocyte infiltration attenuation in the striatum of EAE mice treated with siponimod.

The potential neuroprotective effect of siponimod suggested by these studies could explain the benefit observed on disability progression in patients with SP MS treated with this drug.

In contrast with these findings, the INFORMS trial failed to show a significant effect of fingolimod vs placebo on 3-month confirmed disease progression (measured on a composite end point including EDSS score, 9-hole peg test, and timed 25-foot walk test) in a large population $(n=970)$ of PP MS patients followed for at least 3 years. ${ }^{20}$ Besides the different primary end points of the EXPAND and INFORMS trials, other factors may explain the divergent findings of the two studies. ${ }^{21}$ The EXPAND trial had an adaptive design in 
terms of duration, since the trial was stopped when a predetermined number of events were reached. This methodological strategy might have enhanced the likelihood of detecting an effect of treatment compared to the PP MS fingolimod trial. Other possible explanations of the success of the siponimod trial as opposed to the failure of the fingolimod one in progressive MS are the intrinsic pathogenetic differences between SP and PP MS and the selective interaction of siponimod with types 1 and 5 S1PR, which are known to mediate protective effects on oligodendrocytes, microglia, astrocytes, and neurons. ${ }^{22}$ Overall, S1PR modulators family seems to represent a promising treatment strategy for MS as indicated by the recent findings of ponesimod, ozanimod, and amiselimod Phase II trials in relapsing MS. ${ }^{23-25}$

Publication of final data of the EXPAND trial and further research to confirm the promising results of siponimod and other S1PR modulators studies in MS are warranted.

\section{Disclosure}

The author reports no conflicts of interest in this work.

\section{References}

1. Lorscheider J, Buzzard K, Jokubaitis V, et al. Defining secondary progressive multiple sclerosis. Brain. 2016;139(pt 9):2395-2405.

2. Correale J, Gaitán MI, Ysrraelit MC, Fiol MP. Progressive multiple sclerosis: from pathogenic mechanisms to treatment. Brain. 2017;140(3): 527-546.

3. Reynolds R, Roncaroli F, Nicholas R, Radotra B, Gveric D, Howell O. The neuropathological basis of clinical progression in multiple sclerosis. Acta Neuropathol. 2011;122(2):155-170.

4. Vermersch P, Bar-or A, Cree B, et al. The EXPAND study results: efficacy of siponimod in secondary progressive multiple sclerosis. Eur J Neurol. 2017;24(suppl 1):44.

5. Subei AM, Cohen JA. Sphingosine 1-phosphate receptor modulators in multiple sclerosis. CNS Drugs. 2015;29(7):565-575.

6. Gajofatto A, Turatti M, Monaco S, Benedetti MD. Clinical efficacy, safety, and tolerability of fingolimod for the treatment of relapsing-remitting multiple sclerosis. Drug Healthc Patient Saf. 2015;7:157-167.

7. Pan S, Gray NS, Gao W, et al. Discovery of BAF312 (siponimod), a potent and selective S1P receptor modulator. ACS Med Chem Lett. 2013;4(3): 333-337.

8. Gergely P, Nuesslein-Hildesheim B, Guerini D, et al. The selective sphingosine 1-phosphate receptor modulator BAF312 redirects lymphocyte distribution and has species-specific effects on heart rate. Br J Pharmacol. 2012;167(5):1035-1047.

9. Selmaj K, Li DK, Hartung HP, et al. Siponimod for patients with relapsing-remitting multiple sclerosis (BOLD): an adaptive, dose-ranging, randomised, phase 2 study. Lancet Neurol. 2013;12(8):756-767.
10. Kappos L, Li DK, Stüve O, et al. Safety and efficacy of siponimod (BAF312) in patients with relapsing-remitting multiple sclerosis: dose-blinded, randomized extension of the phase 2 BOLD study. JAMA Neurol. 2016;73(9):1089-1098.

11. Kappos L, Bar-Or A, Cree B, et al. Baseline subgroup characteristics of EXPAND: a phase 3 study of siponimod (BAF312) for the treatment of secondary progressive multiple sclerosis. Neurology. 2016; 86(16 suppl):3.084.

12. Giovannoni G, Bar-or A, Cree B, et al. The EXPAND study results: safety and tolerability of siponimod in patients with secondary progressive multiple sclerosis. Eur J Neurol. 2017;24(suppl 1):495.

13. Kappos L. European Study Group on Interferon beta-1b in Secondary Progressive MS. Placebo-controlled multicentre randomised trial of interferon $\beta-1 b$ in treatment of secondary progressive multiple sclerosis. Lancet. 1998;352:1491-1497.

14. Hartung H-P, Gonsette R, Konig N, et al. Mitoxantrone in progressive multiple sclerosis: a placebo-controlled, double-blind, randomised, multicentre trial. Lancet. 2002;360:2018-2025.

15. Mantia LL, Vacchi L, Rovaris M, et al. Interferon $\beta$ for secondary progressive multiple sclerosis: a systematic review. J Neurol Neurosurg Psychiatry. 2013;84:420-426.

16. Ontaneda D, Fox RJ, Chataway J. Clinical trials in progressive multiple sclerosis: lessons learned and future perspectives. Lancet Neurol. 2015;14(2):208-223.

17. Tourbah A, Lebrun-Frenay C, Edan G, et al. MD1003 (high-dose biotin) for the treatment of progressive multiple sclerosis: a randomised, doubleblind, placebo-controlled study. Mult Scler. 2016;22(13):1719-1731.

18. O'Sullivan C, Schubart A, Mir AK, Dev KK. The dual S1PR1/S1PR5 drug BAF312 (Siponimod) attenuates demyelination in organotypic slice cultures. J Neuroinflammation. 2016;13:31.

19. Gentile A, Musella A, Bullitta S, et al. Siponimod (BAF312) prevents synaptic neurodegeneration in experimental multiple sclerosis. J Neuroinflammation. 2016;13(1):207.

20. Lublin F, Miller DH, Freedman MS, et al. Oral fingolimod in primary progressive multiple sclerosis (INFORMS): a phase 3, randomised, doubleblind, placebo-controlled trial. Lancet. 2016;387(10023):1075-1084.

21. Gajofatto A, Turatti M, Benedetti MD. Primary progressive multiple sclerosis: current therapeutic strategies and future perspectives. Expert Rev Neurother. 2017;17(4):393-406.

22. Chun J, Hartung HP. Mechanism of action of oral fingolimod (FTY720) in multiple sclerosis. Clin Neuropharmacol. 2010;33(2):91-101.

23. Olsson T, Boster A, Fernández Ó, et al. Oral ponesimod in relapsingremitting multiple sclerosis: a randomised phase II trial. $J$ Neurol Neurosurg Psychiatry. 2014;85(11):1198-1208.

24. Cohen JA, Arnold DL, Comi G, et al. Safety and efficacy of the selective sphingosine 1-phosphate receptor modulator ozanimod in relapsing multiple sclerosis (RADIANCE): a randomised, placebo-controlled, phase 2 trial. Lancet Neurol. 2016;15(4):373-381.

25. Kappos L, Arnold DL, Bar-Or A, et al. Safety and efficacy of amiselimod in relapsing multiple sclerosis (MOMENTUM): a randomised, double-blind, placebo-controlled phase 2 trial. Lancet Neurol. 2016; 15(11):1148-1159.

\section{Publish your work in this journal}

Drug Design, Development and Therapy is an international, peerreviewed open-access journal that spans the spectrum of drug design and development through to clinical applications. Clinical outcomes, patient safety, and programs for the development and effective, safe, and sustained use of medicines are the features of the journal, which

\section{Dovepress}

has also been accepted for indexing on PubMed Central. The manuscript management system is completely online and includes a very quick and fair peer-review system, which is all easy to use. Visit http://www.dovepress.com/testimonials.php to read real quotes from published authors. 